
\title{
25 Research Square \\ The Effect of Growth Hormone on In Vitro Fertilization Outcomes During Ovarian Stimulation: A Matched Cohort Study
}

Jillian Kurtz ( $\square$ jillian1 gilbert@gmail.com )

Main Line Fertility https://orcid.org/0000-0002-8190-636X

Nicolle Clements

Saint Joseph's University

Allison Bloom

Main Line Fertility https://orcid.org/0000-0002-5662-0343

John J. Orris

Main Line Fertility

Michael Glassner

Main Line Fertility

Sharon H Anderson

Main Line Fertility

\section{Research Article}

Keywords: Controlled ovarian stimulation, IVF, Growth Hormone, Poor Responder

Posted Date: September 7th, 2021

DOI: https://doi.org/10.21203/rs.3.rs-700354/v1

License: (c) (i) This work is licensed under a Creative Commons Attribution 4.0 International License.

Read Full License 


\section{Abstract}

Background. While growth hormone $(\mathrm{GH})$ is commonly used as an adjuvant treatment to controlled ovarian stimulation (COS) for in vitro fertilization (IVF) cycles, the data regarding its efficacy is inconsistent.

Design. A retrospective matched cohort study of poor responder patients who underwent COS without the use of $\mathrm{GH}(\mathrm{COS}-\mathrm{GH})$ subsequently followed by $\mathrm{COS}$ cycles that included adjuvant $\mathrm{GH}(\mathrm{COS}+\mathrm{GH})$ treatment.

Materials and Methods. A list of all patients having filled a prescription for GH from January 2018 March 2020 was obtained. GH was administered daily at 3mg (9IU) starting on the first day of stimulation and ending on the day of trigger. Only women who had documentation of a previous cycle without the use of $\mathrm{GH}$ were included in the study.

Results. 182 cycles (91 patients) were included in the study, and COS-GH cycles were compared to $\mathrm{COS}+\mathrm{GH}$ cycles. The total dose of gonadotropins used ( 5757 vs $4252 \mathrm{mIU}, \mathrm{p}=0.002)$, duration of stimulation (10.4 vs 10.1 days, $p=-.045$ ), maximum Estradiol (E2) (2411 vs 1932 pg/ml, p=0.010), endometrial thickness (11.2 vs $10.6 \mathrm{~mm}, p=0.010)$, number of oocytes retrieved (14.2 vs $11.8, p=0.001$ ), number of mature oocytes (11.1 vs $9.7, p=0.028$ ), number of blastocysts ( 3.98 vs $2.56, p=<0.001$ ) and number of usable blastocysts ( 2.5 vs $1.6, p=<0.001)$ were all significantly greater in the GH group.

Conclusions. Adding GH to the COS protocol in poor responder patients may lead to improvements in the number of oocytes retrieved, number of mature oocytes, endometrial thickness, number of blastocysts, the number of usable blastocysts.

\section{Introduction}

As women delay childbearing to later in life, the number of women with age-related infertility and the need for assisted reproductive technologies (ART) is on the rise [1]. As a result, the number of women presenting to the IVF center meeting the standard Bologna criteria of poor ovarian response is also on the rise and may be as high as $24 \%$ by some studies [2]. The Bologna criteria is defined as meeting at least two of the following criteria: 1) Age $>40$ or any other risk factor for poor ovarian response, 2) a previous poor ovarian response (<3 oocytes with a conventional stimulation protocol) 3 ) abnormal ovarian reserve test (i.e. antral follicle count $<5-7$ or anti-Mullerian hormone $<0.5-1.1 \mathrm{ng} / \mathrm{ml}$ ) [3]. The need to optimize protocols in poor responder patients is clear, and many centers offer adjuncts in an attempt to improve IVF success rates. Growth Hormone (GH) is one of these clinical adjuncts that has been used in the field of female infertility for the past 25 years [4]. Despite multiple studies on the use of GH as an adjuvant therapy in IVF, its clinical utility remains controversial.

$\mathrm{GH}$ is a peptide hormone secreted from the anterior pituitary in response to growth hormone releasing hormone. It acts primarily through second messengers to increase synthesis of insulin-like growth factor 
1 (IGF-1) by the liver [5]. Zhou et al, demonstrated that IGF-1 acts on granulosa cells of the ovarian follicle via its receptor (IGF-1R) to promote gene expression and potentiate the effects of follicle stimulating hormone (FSH) on the granulosa cells [6]. Growth hormone has been shown in vitro to be an important regulator of ovarian steroidogenesis, follicular development and oocyte maturation [7-9]. Mendoza et al, proved that the oocytes derived from the follicles with the highest concentration of $\mathrm{GH}$ were also the oocytes that led to the embryos with the fastest cleavage and best morphology and thus had the highest transfer rate [10].

A metanalysis published in 2017 by Li, et al on the role of $\mathrm{GH}$ for poor responders undergoing ART showed a statistically significantly higher clinical pregnancy rate and live birth rate with the addition of $\mathrm{GH}$ compared to controls [2]. These results are similar to previous metanalysis however, all suffer the same issue of heterogeneity in study design and small sample sizes overall $[11,12]$. An updated metanalysis by Hart et al, also published in 2017 but included the LIGHT study found an increase in several clinical parameters but no increase in live birth rate $[13,14]$. As highlighted in this metanalysis, due to the low live birth rate in poor responder patients, the number required for power is substantial and many studies lack the sufficient number of subjects to draw large conclusions regarding live birth rate. Several studies have since been published with inconsistent results [15-19]. Because of the inconsistencies in study findings, both ASRM and ESHRE have failed to support the universal use of GH in the ovarian stimulation protocol of poor responder patients [20,21]. The purpose of this study is to evaluate the role of $\mathrm{GH}$ as an adjuvant treatment in women with a history of a poor IVF outcome.

\section{Materials And Methods}

This retrospective study was conducted out of a single IVF center located in Bryn Mawr, Pennsylvania. A list of all patients having obtained a prescription for GH from January 2018 to March 2020 was obtained. All patients underwent stimulation with the same provider. Most patients followed a standard antagonist protocol with a human chorionic gonadotropin (hCG) trigger when appropriate.

To allow for a matched study where every patient served as their own control, patients were included in the study only if they had record of a previous stimulation cycle in which $\mathrm{GH}$ was not utilized to allow for comparison between cycles. The age and $\mathrm{AMH}$ were therefore the same in the two cohorts, 36 years and $2.3 \mathrm{ng} / \mathrm{ml}$ respectively. This design allowed for elimination of several variables and confounders which were present in other studies $[11,12]$.

Patients were excluded if the cycle was cancelled prior to retrieval or if sperm was obtained via surgical extraction. A total of 91 patients met criteria for participation. Infertility diagnoses included diminished ovarian reserve, ovulation dysfunction as well as tubal factor infertility. GH was administered daily at $3 \mathrm{mg}$ (9 IU) starting on the first day of stimulation and ending on the day of hCG trigger. Because this study was a retrospective review of deidentified patient information an exemption from the IRB was granted. 


\section{Results}

The characteristics of a patient's IVF cycle without GH were compared to their IVF cycle with GH. A paired t-tests was run on all 91 patients using 11 variables. To correct for any Type I error, the alpha threshold was lowered. The results are summarized in the table below in Table 1.

The total dose of gonadotropins used ( 5757 vs $4252 \mathrm{mlU}, \mathrm{p}=0.002)$, duration of stimulation (10.4 vs 10.1 days, $p=-.045)$, $\max E 2$ (2411 vs $1932 \mathrm{pg} / \mathrm{ml}, \mathrm{p}=0.010)$, endometrial thickness (11.2 vs $10.6 \mathrm{~mm}, \mathrm{p}=0.010)$, number of oocytes retrieved (14.2 vs $11.8, p=0.001)$, number of mature oocytes (11.1 vs $9.7, p=0.028$ ), number of blastocysts (3.98 vs $2.56, p=<0.001)$ and number of usable blastocysts ( 2.5 vs $1.6, p=<0.001)$ were all significantly greater in the GH group. Usable blastocysts were defined as blastocysts that are high enough quality for transfer or cryopreservation. At this facility, only embryos that are designated as A or B according to the Gardner embryo grading system are transferred or cryopreserved. All embryos given a $\mathrm{C}$ designation are discarded. The two variables of greatest clinical significance (blastocysts and usable blastocysts) are plotted in Figure 1 below.

A secondary analysis was performed to evaluate if $\mathrm{GH}$ had an impact on euploid rate. In the group without $\mathrm{GH}, 35$ cycles elected for preimplantation genetic testing for aneuploidy (PGT-A). Of those embryos that were biopsied there was an overall $35.09 \%$ euploidy rate. In the group with $\mathrm{GH}, 44$ cycles elected for PGT-A and of those embryos that were biopsied there was an overall euploidy rate of $51.3 \%$ $(p=0.0158)$.

Importantly, the GH and non-GH patients who elected to undergo PGT-A did not differ significantly in their ages or AMH levels. The 35 non-GH patients had an average age 35.74 and an average AMH $2.87 \mathrm{ng} / \mathrm{ml}$. The $44 \mathrm{GH}$ patients had an average age of $36.22(p=0.5458)$ and average AMH $2.72 \mathrm{ng} / \mathrm{ml}(\mathrm{p}=0.79)$.

\section{Discussion}

The use of GH as an adjunct to IVF has been studied extensively with conflicting results. As the number of patients seeking out ART that are older or fall into the category of poor responder patients increase, the need to optimize protocols and understand the importance of adjunctive treatment is critical. Our study findings revealed that co-treatment with $\mathrm{GH}$ in women with a history of poor ovarian response in prior cycle could improve the endometrial thickness, increase the number of oocytes retrieved, increase the number of mature oocytes, increase the number of blastocysts, increase the number of usable blastocysts and also increase the percentage of euploid blastocysts. This finding supports the metanalysis published by Li, et al in 2017 [2]. It also corroborates the more recent findings by Li and Gong in their randomized controlled trials to look at GH use in the setting of history of poor embryo development and poor responder patients respectively. Both studies supported the use of GH in IVF cycles $[15,17]$. This is contrast to the findings by Zhu, et al who found that live birth rate was not improved with the use of GH as adjuvant treatment. They did however, find an increase in Day 3 embryo quality [19]. 
One strength of the study is in the design. Utilizing patients as their own control in evaluating how the addition of GH changed cycle parameters eliminates some confounding factors present in previously published studies [11,12]. However, because of the nature of the study design pregnancy outcomes could not be evaluated. A poor outcome in the non-GH group was the incentive to proceed with a subsequent IVF cycle in which GH was utilized as an adjunct. We can assume, however, that an increased number of high-quality blasts, particularly euploid blasts, may translate to an increase in live birth rate per transfer [22]. This is an area of future study.

Many studies that have previously evaluated GH in controlled ovarian stimulation included patients that meet the Bologna criteria for poor ovarian response. As outlined in the introduction, the Bologna criteria helps define poor responder patients [3]. Interestingly, our average study patient was age 36 years with an $\mathrm{AMH}$ of $2.3 \mathrm{ng} / \mathrm{ml}$ which does not satisfy the traditional criteria of poor responder patients as defined by these criteria. This suggests that GH may benefit a larger range of patients and should be considered in anyone with history of poor embryo development. A recent study by Li et al, does suggest that $\mathrm{GH}$ supplementation improves oocyte competence in women with a history of poor embryonic development [15].

Another interesting finding is the total dose of gonadotropins used in the cycles. We know from previous studies that GH supplementation induces FSH receptor expression and should, in theory, sensitize the patient to exogenous gonadotropins [24]. This has been shown to be true in several other studies in which adding growth hormone to the protocol resulted in a lower required dose of gonadotropins [2]. Conversely, in our study, a higher dose of gonadotropins was used in the cycles with the addition of $\mathrm{GH}$. This was due to provider preference. Despite this confounder, the results suggest there may be a role for growth hormone in the ovarian stimulation protocol in women who have had poor outcomes in previous cycles.

To complicate matters, there is no accepted dose or timing of administration therefore a great deal of heterogeneity exists between studies in regard to these two parameters. In the present study, we administered 3mg (9IU) per day of stimulation. The dosing is consistent with Li et al. though they did not start until the day of downregulation in antagonist cycles [14]. Bassiouny and Bayoumi used a slightly lower dose of $2.5 \mathrm{mg} /$ day, though they did not start until day 6 of stimulation [24,25]. Safderin had an arm receive $2.5 \mathrm{mg} /$ day as well though this did not start until day 8 and another arm that received $0.1 \mathrm{mg} /$ day from cycle day 3 of the previous cycle [18]. Gong et al, administered 4IU/day (1.3mg) starting from Day 4 of the previous cycle [17]. In the LIGHT study, $12 \mathrm{IU} /$ day $(4 \mathrm{mg})$ from day of stimulation [14]. The variability in dosing regimens across studies makes it difficult to interpret results.

\section{Conclusion}

Adding Growth Hormone to the controlled ovarian stimulation protocol of poor responder patients leads to improvements in the number of oocytes retrieved, number of mature oocytes, endometrial thickness, number of blastocysts, number of usable blastocysts and the percent of euploid blastocysts. 


\section{Declarations}

Acknowledgements: Portions of this data have been presented at the ASRM conference in 2019 and have been published as an abstract with Fertility and Sterility. The complete manuscript that follows is original and has never been published elsewhere.

- Funding: Not Applicable

- Conflicts of Interest/competing interests: Not Applicable

- Availability of Data and Material: Data available on request from the authors

- Code Availability: Not Applicable

- Author's Contributions: JK conceived the original idea, collected the data and took the lead on writing the manuscript. NC performed the statistical analysis. SA supervised the project and contributed to the manuscript. All authors discussed the results and commented on the manuscript.

- Ethics Approval: IRB exemption attached

- Consent to participate: Not applicable

- Consent for publication: Not applicable

\section{Conflict of Interest Statement}

\section{The Effect of Growth Hormone on IVF outcomes during Ovarian Stimulation: a Matched Cohort Study}

The authors whose names are listed immediately below certify that they have NO affiliations with or involvement in any organization or entity with any financial interest (such as honoraria; educational grants; participation in speakers' bureaus; membership, employment, consultancies, stock ownership, or other equity interest; and expert testimony or patent-licensing arrangements), or non-financial interest (such as personal or professional relationships, affiliations, knowledge or beliefs) in the subject matter or materials discussed in this manuscript.

Jillian Kurtz

Sharon Anderson

Nicolle Clements

Allison Bloom

Michael Glassner

John Orris

\section{References}


1. Matthews TJ, Hamilton BE (2009) Delayed childbearing: more women are having their first child later in life. NCHS Data Brief. 21:1-8. PMID: 19674536.

2. Li XL, Wang L, Lv F, Huang XM, Wang LP, Pan Y, Zhang XM (2017) The influence of different growth hormone addition protocols to poor ovarian responders on clinical outcomes in controlled ovary stimulation cycles: A systematic review and meta-analysis. Medicine (Baltimore). 96(12):e6443. doi: 10.1097/MD.0000000000006443. PMID: 28328856; PMCID: PMC5371493.

3. Younis JS, Ben-Ami M, Ben-Shlomo I (2015) The Bologna criteria for poor ovarian response: a contemporary critical appraisal. J Ovarian Res. 8:76. doi: 10.1186/s13048-015-0204-9. PMID: 26577149; PMCID: PMC4650906.

4. Hart RJ (2019) Use of Growth Hormone in the IVF Treatment of Women with Poor Ovarian Reserve. Front Endocrinol (Lausanne). 10:500. doi: 10.3389/fendo.2019.00500. PMID: 31396160; PMCID: PMC6667844.

5. Xu YM, Hao GM, Gao BL (2019). Application of Growth Hormone in in vitro Fertilization. Front Endocrinol (Lausanne). 10:502. doi:10.3389/fendo.2019.00502

6. Zhou P, Baumgarten SC, Wu Y, Bennett J, Winston N, Hirshfeld-Cytron J, Stocco C (2013) IGF-I signaling is essential for FSH stimulation of AKT and steroidogenic genes in granulosa cells. Mol Endocrinol. 27(3):511-23. doi: 10.1210/me.2012-1307. PMID: 23340251; PMCID: PMC3589673.

7. Bachelot A, Monget P, Imbert-Bolloré P, Coshigano K, Kopchick JJ, Kelly PA, Binart N (2002). Growth hormone is required for ovarian follicular growth. Endocrinology. Oct;143(10):4104-12. doi: 10.1210/en.2002-220087. PMID: 12239122.

8. Nakamura E, Otsuka F, Inagaki K, Miyoshi T, Matsumoto Y, Ogura K, Tsukamoto N, Takeda M, Makino $H$ (2012). Mutual regulation of growth hormone and bone morphogenetic protein system in steroidogenesis by rat granulosa cells. Endocrinology. 153(1):469-80. doi: 10.1210/en.2011-1646. Epub 2011 Nov 8. PMID: 22067323.

9. Bevers MM, Izadyar F. Role of growth hormone and growth hormone receptor in oocyte maturation (2002) Mol Cell Endocrinol. 197(1-2):173-8. doi: 10.1016/s0303-7207(02)00270-8. PMID: 12431810.

10. Mendoza C, Ruiz-Requena E, Ortega E, Cremades N, Martinez F, Bernabeu R, Greco E, Tesarik J (2002) Follicular fluid markers of oocyte developmental potential. Hum Reprod. 17(4):1017-22. doi: 10.1093/humrep/17.4.1017. PMID: 11925399.

11. Kolibianakis EM, Venetis CA, Diedrich K, Tarlatzis BC, Griesinger G (2009) Addition of growth hormone to gonadotrophins in ovarian stimulation of poor responders treated by in-vitro fertilization: a systematic review and meta-analysis. Hum Reprod Update. 15(6):613-22. doi: 10.1093/humupd/dmp026. Epub 2009 Jun 26. PMID: 19561136.

12. Duffy JM, Ahmad G, Mohiyiddeen L, Nardo LG, Watson A (2010) Growth hormone for in vitro fertilization. Cochrane Database Syst Rev.1:CD000099. doi: 10.1002/14651858.CD000099.pub3. PMID: 20091500; PMCID: PMC7058116.

13. Hart R, Rombauts L, Norman RJ (2017) Growth Hormone in IVF cycles: any hope? Current Opinion in Obstetrics and Gynecology. 29(3):119-125 doi: 10.1097/GC0.0000000000000360 
14. Norman RJ, Alvino H, Hull LM, Mol BW, Hart RJ, Kelly TL, Rombauts L; LIGHT investigators (2019) Human growth hormone for poor responders: a randomized placebo-controlled trial provides no evidence for improved live birth rate. Reprod Biomed Online. 38(6):908-915. doi: 10.1016/j.rbmo.2019.02.003. Epub 2019 Feb 27. PMID: 30954433.

15. Li J, Chen Q, Wang J, Huang G, Ye H (2020). Does growth hormone supplementation improve oocyte competence and IVF outcomes in patients with poor embryonic development? A randomized controlled trial. BMC Pregnancy Childbirth. 20(1):310. doi: 10.1186/s12884-020-03004-9. PMID: 32434490; PMCID: PMC7238549.

16. Choe SA, Kim MJ, Lee HJ, Kim J, Chang EM, Kim JW, Park HM, Lyu SW, Lee WS, Yoon TK, Kim YS (2017) Increased proportion of mature oocytes with sustained-release growth hormone treatment in poor responders: a prospective randomized controlled study. Arch Gynecol Obstet. 297(3):791-796. doi: 10.1007/s00404-017-4613-4. PMID: 29264647.

17. Gong Y, Zhang K, Xiong D, Wei J, Tan H, Qin S (2020). Growth hormone alleviates oxidative stress and improves the IVF outcomes of poor ovarian responders: a randomized controlled trial. Reprod Biol Endocrinol. 18(1):91. doi: 10.1186/s12958-020-00648-2. PMID: 32891163; PMCID: PMC7487463.

18. Safdarian L, Aghahosseini M, Alyasin A, Samaei Nouroozi A, Rashidi S, Shabani Nashtaei M, Najafian A, Lak P (2019) Growth Hormone (GH) Improvement of Ovarian Responses and Pregnancy Outcome in Poor Ovarian Responders: A Randomized Study. Asian Pac J Cancer Prev. 20(7):20332037. doi: 10.31557/APJCP.2019.20.7.2033. PMID: 31350962; PMCID: PMC6745232.

19. Zhu J, Wang Y, Chen L, Liu P, Li R, Qiao J (2020) Growth Hormone Supplementation May Not Improve Live Birth Rate in Poor Responders. Front Endocrinol (Lausanne). 11:1. doi:

10.3389/fendo.2020.00001. PMID: 32038495; PMCID: PMC6990136.

20. Ovarian Stimulation TEGGO, Bosch E, Broer S, Griesinger G, Grynberg M, Humaidan P, Kolibianakis E, Kunicki M, La Marca A, Lainas G, Le Clef N, Massin N, Mastenbroek S, Polyzos N, Sunkara SK, Timeva T, Töyli M, Urbancsek J, Vermeulen N, Broekmans F (2020) ESHRE guideline: ovarian stimulation for IVF/ICSI ${ }^{\dagger}$. Hum Reprod Open. doi: 10.1093/hropen/hoaa009. PMID: 32395637; PMCID: PMC7203749.

21. Kamath MS, Mascarenhas M, Franik S, Liu E, Sunkara SK (2019) Clinical adjuncts in in vitro fertilization: a growing list. Fertil Steril. 112(6):978-986. doi: 10.1016/j.fertnstert.2019.09.019. PMID: 31703943.

22. Adamson GD, de Mouzon J, Chambers GM, Zegers-Hochschild F, Mansour R, Ishihara O, Banker M, Dyer S. International Committee for Monitoring Assisted Reproductive Technology: world report on assisted reproductive technology, 2011 (2018) Fertil Steril. 2018 Nov;110(6):1067-1080. doi: 10.1016/j.fertnstert.2018.06.039. PMID: 30396551.

23. Regan SLP, Knight PG, Yovich JL, Arfuso F, Dharmarajan A (2018) Growth hormone during in vitro fertilization in older women modulates the density of receptors in granulosa cells, with improved 
pregnancy outcomes. Fertil Steril. 110(7):1298-1310. doi: 10.1016/j.fertnstert.2018.08.018. PMID: 30503129.

24. Bassiouny YA, Dakhly DMR, Bayoumi YA, Hashish NM (2016) Does the addition of growth hormone to the in vitro fertilization/intracytoplasmic sperm injection antagonist protocol improve outcomes in poor responders? A randomized, controlled trial. Fertil Steril. 105(3):697-702. doi: 10.1016/j.fertnstert.2015.11.026. PMID: 26690008.

25. Bayoumi YA, Dakhly DM, Bassiouny YA, Hashish NM (2015) Addition of growth hormone to the microflare stimulation protocol among women with poor ovarian response. Int J Gynaecol Obstet. 131(3):305-8. doi: 10.1016/j.ijgo.2015.05.034.

\section{Tables}

Table 1: The characteristics of a patient's IVF cycle without GH were compared to their IVF cycle with GH. A paired t-tests was run on all 91 patients using 11 variables. To correct for any Type I error, the alpha threshold was lowered.

\begin{tabular}{llll} 
Averages (SD) & Growth Hormone Present? & \\
\hline Variable & Yes & No & p-value \\
\hline Total Dose & $4757(1925)$ & $4252(1785)$ & 0.002 \\
\hline Duration of Stimulation & $10.44(1.85)$ & $10.09(1.62)$ & 0.045 \\
\hline Endometrial Thickness & $11.21(2.33)$ & $10.69(1.95)$ & 0.01 \\
\hline Max E2 & $2411(1352)$ & $1932(959)$ & $<0.0001$ \\
\hline Max P4 & $0.854(0.459)$ & $0.935(0.544)$ & 0.928 \\
\hline \# of Follicles on Ovaries & $12.66(7.09)$ & $11.81(6.64)$ & 0.063 \\
\hline \# Oocytes & $14.25(9.08)$ & $11.80(6.82)$ & 0.001 \\
\hline Total MII (mature eggs) & $11.13(7.19)$ & $9.74(6.59)$ & 0.028 \\
\hline \# Blastocyst Embryos & $3.99(3.70)$ & $2.56(2.53)$ & $<0.0001$ \\
\hline \# Usable Blastocysts & $2.52(2.5)$ & $1.68(1.74)$ & $<0.0001$ \\
\hline \% Usable Blastocysts & $30.74(25.91)$ & $27.69(25.65)$ & 0.154
\end{tabular}

\section{Figures}





\section{Figure 1}

The total dose of gonadotropins used ( 5757 vs $4252 \mathrm{mlU}, \mathrm{p}=0.002)$, duration of stimulation (10.4 vs 10.1 days, $p=-.045)$, $\max E 2$ (2411 vs $1932 \mathrm{pg} / \mathrm{ml}, \mathrm{p}=0.010)$, endometrial thickness (11.2 vs $10.6 \mathrm{~mm}, \mathrm{p}=0.010$ ), number of oocytes retrieved (14.2 vs $11.8, p=0.001)$, number of mature oocytes (11.1 vs $9.7, p=0.028)$, number of blastocysts (3.98 vs $2.56, p=<0.001)$ and number of usable blastocysts $(2.5$ vs $1.6, p=<0.001)$ were all significantly greater in the GH group. Usable blastocysts were defined as blastocysts that are 
high enough quality for transfer or cryopreservation. At this facility, only embryos that are designated as A or B according to the Gardner embryo grading system are transferred or cryopreserved. All embryos given a $\mathrm{C}$ designation are discarded. The two variables of greatest clinical significance (blastocysts and usable blastocysts) are plotted 